\title{
Memory load and latency in recognition of pictures*
}

\author{
WILLIAM P. BANKS $\dagger$ \\ Pomona College. Claremont. California 91711 \\ and \\ GRIFFIN R. FARIELLO \\ Stanford University', Stanford. California 94305
}

\begin{abstract}
Color photographs of common scenes were used as memory and test items in two memory search experiments. Memory load varied from 2 to 24 items in one experiment and from 4 to 64 in the other. Latency of classification of test items increased with memory load. The increase is very close to linear for loads up to six items, but for the full range of memory loads, it is negatively accelerated and approximately logarithmic. Sternberg's (1966, 1969) serial theory of memory search, which predicts linear functions, holds for small memory loads of pictures. If serial search takes place for large memory loads, the speed of search must be faster than for small loads. It is also possible that items in memory loads of all sizes are accessed by a single process that generates a negatively accelerated relation between size of load and latency.
\end{abstract}

In Sternberg's $(1966,1969)$ memory search paradigm, the $\mathrm{S}$ classifies singly presented probe items as members or nonmembers of a short, previously memorized list. This paradigm frequently gives a linear increasing relation between reaction time (RT) to classify probes and size of the memorized list (s). The linear increase in RT with s led Sternberg to formulate a serial model of memory search in which the probe is first encoded and then is compared to the members of the memory set, item by item. When a linear equation is fit to the empirical relation between mean RT and $s$, the $y$ intercept of the equation is the sum of constant latencies such as probe identification time and response selection, and the slope of the function equals the memory search rate.

Although Sternberg's own functions appear to be irsolutely linear and inconsistent with anything but a strictly serial search model (see especially Sternberg, 1966, Figs. 1 and 2), there exists some controversy on the question. Not uncommonly, researchers find a negatively accelerated increase in $\mathrm{RT}$ with $\mathrm{s}$ that is more nearly logarithmic than linear. Nickerson, (1972, pp. 279-280) reviews some experiments relevant to this issue. He makes the point that, when the independent variable ( $\mathrm{s}$, in this case) takes on a range of values as limited as is usually the case in memory search

*This research was supported by a Pomona Coilege research grant to William P. Banks. Both experiments were performed at Stanford University, and the authors are grateful to the Psychology Department and to Professor E.E. Smith for providing facilities and apparatus for performing the experiments. R. C. Atkinson, D. J. Herrmann, S. M. Kosslyn, and E. Tulving provided stimulating comments at various stages of this research. The authors thank D. Berger for a careful critical reading of a draft of this paper.

$\uparrow$ Requests for reprints should be sent to William P. Banks, Department of Psychology, Pomona College, Claremont, California 91711. experiments, it may be difficult to distinguish between a logarithmic and a linear function.

In the two experiments reported here, the memory load of pictures varies from 2 to 24 and from 4 to 64 items. While the wide range of set sizes in these experiments satisfied Nickerson's point about the range of $s$, these experiments cannot really answer the question of linearity of memory search in Sternberg's model because his model refers to short-term ("active") memory. Sets larger than 5 or 10 could be assumed to be stored in long-term memory and. thus, be outside the domain of the serial model. Nevertheless, it is of interest to investigate large memory set sizes, if only to determine the limits of Sternberg's model.

A number of previous experiments have investigated memory set sizes larger than the immediate memory span (e.g., Wingfield \& Branca, 1970; Juola, Fischler, Wood, \& Atkinson, 1971; Atkinson \& Juola, 1973), but these studies have frequently had error rates high enough (10\% to $20 \%$ ) to complicate conclusions about latencies. Also, some of these studies (e.g., Juola et al, 1971) have had different groups of Ss for each memory set size and required each $S$ to memorize one list of words prior to the experimental session. With this procedure, strategy differences between Ss who memorized long and short lists could obscure effects of list length on RT. To avoid this possibility, it would be necessary to have all Ss perform at all levels of memory set size. However, even when the same Ss performed at all levels of memory load, there could still be strategy differences in storage and/or retrieval between long and short lists because of the rehearsal and practice required for long but not for short lists

Photographs of common scenes provide a type of stimulus material for which recognition is extremely accurate even with very large memory sets whose members were briefly presented during learning 


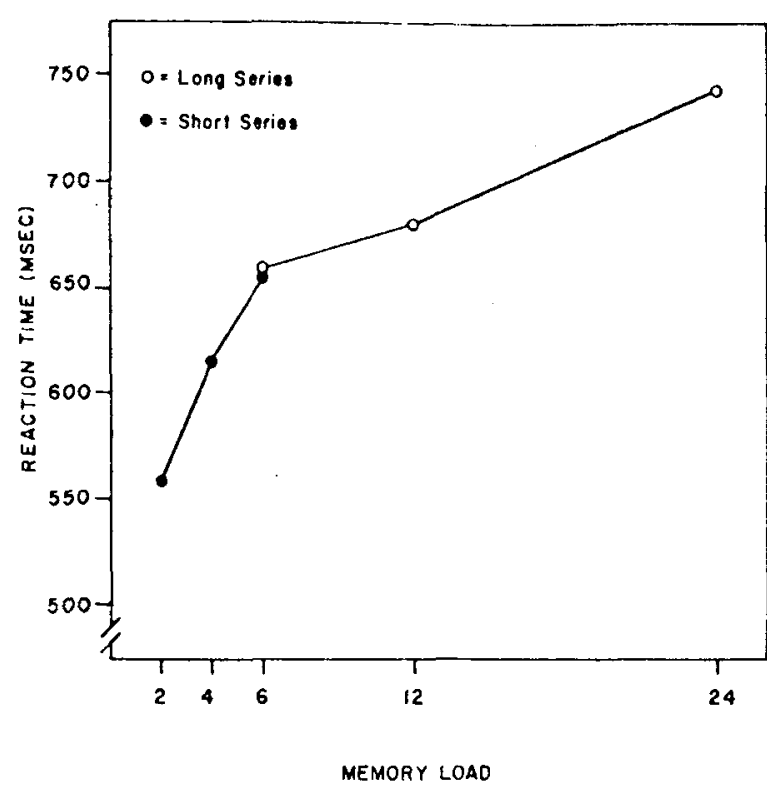

Fig. 1. Mean RT to classify probes as a function of memory load: Experiment 1 .

(Shepard, 1967; Standing, Conezio, \& Haber, 1970). Thus, with pictures as stimuli, it is possible to have very large memory sets, yet use the same procedure for presenting items in long and short sets. In the present experiments, memory sets were color photographs, and the Ss saw each member of the memory set once for $10 \mathrm{sec}$. They did not know how large the memory set was on any given trial until all pictures had been presented. Presumably, then, variations in latency with memory load will reflect differences among the loads only in search processes, not storage strategies.

\section{EXPERIMENT 1}

This experiment had two parts, a short series in which $s=2,4$, or 6 pictures and a long series in which $s=6$, 12 , or 24 pictures. The short series was used to make sure that memory sets composed of photographs would give a linear increase in RT with $s$ up to $s=6$, as is the case for letters and digits. The long set extended the range of $s$.

\section{Method}

A pool of 300 slides of diverse, easily identifiable scenes was selected, with some care taken to minimize similarities of all kinds among the pictures. Memory sets of 2, 4, and 6 items for the short series and 6,12 , and 24 for the long series, as well as the distractors and practice items, were sampled without replacement from this pool.

The short and long series were administered in two separate blocks, with half the Ss having the long series first and half having the short series first. Before testing began, Ss were given practice on memory sets of 3,5 , and 15 items. In both series, memory set items were shown at a rate of $10 \mathrm{sec} /$ picture with a Carousel slide projector which front projected a $1 \times 1 \mathrm{~m}$ image about $3 \mathrm{~m}$ from the $\mathrm{S}$. An externally operated leaf shutter controlled the exposure duration. After the last slide in the memory set, $E$ told $S$ that testing would begin, and the first probe was presented about $5 \mathrm{sec}$ later. Probes werc presented with the same Carousel system used for the memory items. and they remained in view until the $S$ responded. The response was indicated by throwing one of two microswitches: "Yes" responses were aiways executed with the left hand and "no" with the right. The RT was measured from the onset of the probe. Each memory set in the short series was tested with six probes before the next set was presented, and each long series set was tested with 10 probes. Positive and negative probes were used equally often, serial positions in the memory set were equally sampled by the positive probes, and negative probes were never repeated. For $s=2$ in the short series, one positive item was probed twice and, when this happened, the two identical probes were separated by two or more trials.

There were three different sets of pictures for each memory set size in both long and short series, and each $S$ went through nine memory sets in each series in a counterbalanced order. The multiple tests of each memory set, plus the duplication of memory sets, generated nine positive and nine negative probes for each value of $s$ for each $S$ in the short series and 15 positive and 15 negative probes in the long series.

Ss were 12 right-handed female Stanford University undergraduates serving for $1 \mathrm{~h}$ to fulfill a course requirement. They received bonus pay of up to $\$ 2$, from which 50 cents was deducted for every error.

\section{Results and Discussion}

Mean RT for correct responses as a function of $s$ is shown in Fig. 1. Yes and no responses were averaged for this figure because analysis of variance revealed that neither the main effect of type of response nor its interactions were significant. The increase in RT with $\mathrm{s}$ is highly significant, with a $F(5,50)$ of $14.5, p<.001$.

Viewed separately, the long and short series each appear to give a linear increase in RT as $\mathrm{s}$ increases. The linearity of the two series is apparent in Fig. 1, which is plotted in linear coordinates, and a linear least-squares regression equation fit to the two series separately accounts for $99 \%$ of the variance of the means in both cases. However, the two series gave rather different linear functions: The slope was $24 \mathrm{msec}$ per item for the short series, but only $5 \mathrm{msec}$ per item for the long series, and intercepts were $510 \mathrm{msec}$ for the short and 626 for the long series.

A speed-accuracy tradeoff cannot account for the slope differences between the two series. Errors were below $1 \%$ for all memory loads except for $\mathrm{s}=24$, where there were $4 \%$ errors, and the latency for $\mathrm{s}=24$ falls above the regression line for the long series and clearly does not, by itself, account for the lower estimated slope in the long series.

A possible explanation of the differences between the long and short series is provided by Atkinson and Juola's model of recognition memory (Juola, Fischler, Wood, \& Atkinson, 1971; Atkinson \& Juola, 1973). According to this model, items stored for memory search can be either in a short-term or a long-term store. Search rate is faster in the long-tern store than in the short-term store. Presumably, in this experiment, Ss stored and searched the memory loads in the short series in a short-term store and the loads in the long series in a long-term store. The fact that the long and short series were administered it separate blocks makes it possible that $\mathrm{Ss}$ could have 
different strategies for the two ranges of memory load, although it seems unlikely that the two strategies would result in the same $\mathrm{RT}$ for $\mathrm{s}=6$.

Another possibility is that memory sets of all sizes are searched in a single store by a parallel process that terminates after all memory items have been examined. If the completion times are distributed exponentially, the finishing time for the last comparison and, hence, for the entire comparison process will increase linearly with the logarithm of the number of items in memory (Rapoport, 1959). (Other types of search models will also predict negatively accelerated functions. See, for example, Townsend, 1971; Welford, 1960.) ${ }^{1}$ The advantage of this possibility is parsimony. Only one memory store and a single search model for all loads is required. A logarithmic function does fit the data very well. The best fitting $\log$ function (RT $=517+50 \log _{2} \mathrm{~s}$ ) for the range of $s$ from 2 to 24 accounts for $97 \%$ of the variance of the means, while the best linear function fit to the full range accounts for only $82 \%$ of the variance of the means. The nonlinear component of the memory load effect is significant beyond the $p<.01$ level, with an $F(4,40)$ of 8.81 .

A separate analysis was based on the response to the first probe for each memory set rather than on the average of the responses. The results of this analysis are very similar to the results of the analysis of all responses, and both analyses lead to the same conclusions. It is important that the RT vs s relation observed here is not simply a consequence of the repeated testing (cf. Juola et al, 1971).

\section{EXPERIMENT II}

This experiment extends the range of memory loads and tests the simple dual-store serial search model that fits the results of Experiment I. When memory load varied from 2 to 24 items, the two-store serial model could fit the overall curvilinear trend of the RT vs $s$ function by assuming a switch from short-term to long-term memory search at about six items. In this experiment, memory load takes on the values $4,24,44$, 64 items. The two-store model, unembellished by additional assumptions, does not predict a decelerated function beyond 24 items.

\section{Method}

Memory sets and distractors were sampled without replacement from the pool of pictures used in Experiment I. There were two different memory sets of each of the four lengths $(4,24,44$, and 64$)$, making a total of eight different memory sets. After practice loads of 10 and 25 , each $S$ went through the eight in a different random order with the restriction that the first four memory sets (and hence the last four) were of different sizes. After half of the Ss had been tested, the pictures were shuffled and resorted into new target and distractor sets for the second half of the Ss.

The projecting and timing system of Experiment I was used. Presentation of memory sets was at a $10 \mathrm{sec} /$ item rate. Each memory set was tested with five positive and five negative

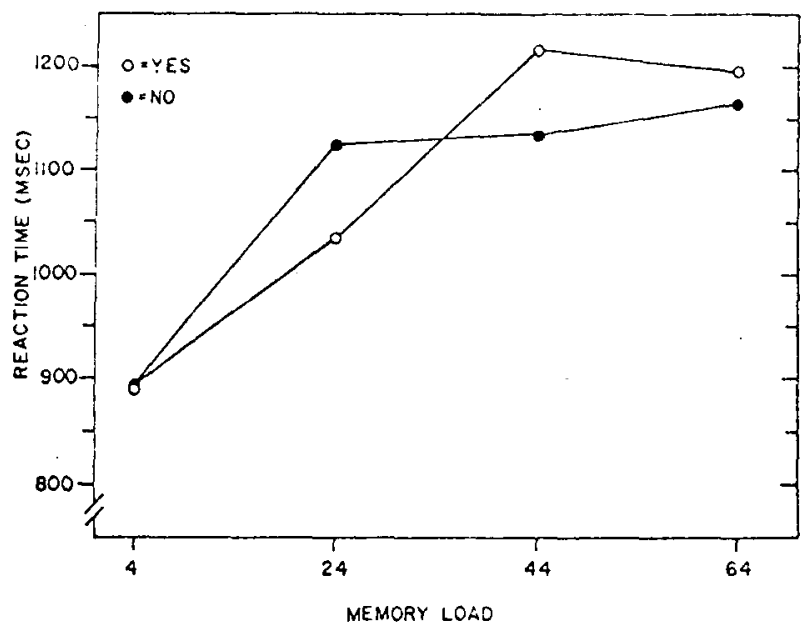

Fig. 2. Mean RT for positive (yes) and negative (no) classification probes as a function of memory load: Experiment II.

probes. Negative probes were never repeated. Positive probes were sampled fairly evenly from all serial positions of the memory set and were separately selected for each S. No memory set items were probed twice except for $s=4$, and the two tests of the same item in this set were always separated by at least two probes.

Ss were 18 male and female Stanford University undergraduates serving for $\$ 2 / \mathrm{h}$ plus $\$ 2.50$ bonus pay from which 50 cents was deducted for every error. Four additional Ss were rejected from the experiment because they made more than five errors.

\section{Results and Discussion}

Mean RT for correct responses is plotted as a function of $s$ in Fig. 2. The increase in RT with $s$ is highly significant, with a $F(3,51)$ of $38.1, p<.001$. Yes and no functions of $s$ are quite different, and the interaction of Type of Response by Memory Load is significant, with an $F(3,51)$ of $3.9, p<.025$. The overall increase in RT with $\mathrm{s}$ has a negatively accelerated trend. The nonlinear component of the memory load effect is significant, with $F(2,28)=9.9, p<.001$.

The function $\mathrm{RT}=740+75 \log _{2} \mathrm{~s}$, where the numbers are in milliseconds, provides an excellent fit to the data, with $r^{2}=99$. A linear function fit to the same data gives an $r^{2}$ of 84 . When only the RTs for $s=24$, 44 , and 64 are considered, a $\log$ function ( $R T=758+$ $72 \log _{2} s$ ) still provides a good fit to the data, with an $r^{2}=86$, while the $r^{2}$ for the best linear function is .77 . However, the nonlinear component of the RT vs memory load function does not reach significance for the loads of 24,44 , and 64 $[F(1,14)=3.3, .1<p<.05]$

These results show that the single-store serial search model ceases to hold at least by the time memory load has reached 24 items. A serial search model with two memory stores cannot handle the results either, unless the switch between long-term and short-term search is assumed to take place at a memory load somewhere 
between 24 and 44 items. Surely, this would be an ad hoc assumption, and one inconsistent with the results of Experiment I. The multistore model of Atkinson and Juola can, however, accommodate these results. Central to their model is a provision for "fast yes" and "fast no" responses that are based on a familiarity judgment of the probe and are executed without a list search. The mean RT for classification of a probe as a member or nonmember of the memory set is, according to this theory, the weighted average of the RT in trials when a "fast" response was made and the RT on trials when a search was conducted, with the weights being the probabilities of the two kinds of response. If the probability of a fast yes or no response increases with memory load, then the RT vs s function will be negatively accelerated.

One index of the proportion of "fast" responses is the error rate, because the fast responses are more errorful than those based on memory search. The error rates are in agreement with what the Atkinson and Juola model would predict. For memory loads of $4,24,44$, and 64 , they are $0.00 \%, 0.3 \%, 2.1 \%$, and $5.6 \%$, respectively. However, it is possible that these errors do not actually represent the "fast" responses of the Atkinson-Juola model. First, every one of these errors was a false negative (response was "no" to a positive probe), and even the four rejected Ss went along with this trend, making only one false positive response among them. It seems likely, therefore, that these errors result from failure of storage rather than fast no responses. The fact that most Ss objected strenuously to being counted wrong for their false negative errors also suggests that the errors resulted from nonstorage of some of the pictures. Some Ss even demanded proof at the end of the experiment that the picture in question was actually shown in the memory set. Of course, Ss stood to lose 50 cents for each error and might be expected to object to being counted wrong.

Another argument against these error responses being familiarity-based fast no responses is based on the RTs. According to the Atkinson-Juola model, the familiarity-based responses, whether they are wrong or right, should always have the same RT, regardless of memory load, because they are all initiated prior to memory search. However, error RTs increased with memory load. There were no errors for $\mathrm{s}=4$ but for $\mathrm{s}=$ 24,44 , and 64 , the error RT means were 1185,1365 , and $1820 \mathrm{msec}$, respectively. It seems likely that the majority of these error responses are not fast nos but issue at the end of an errorful memory comparison process.

The very first responses made by each $S$ to the positive and negative probes for each set size were given the same analyses that the average responses had. In Experiment I virtually the same results were obtained for first and average responses, but, in Experiment 11 . the first response with the memory load of four was much slower than the average response with that memory load (962 vs 889 msec). The RTs for probes presented under the other memory loads in Experiment II were about the same for first and average responses. It would seem that repeated testing facilitated performance more for the memory load of four than for the others. The slower RT for $\mathrm{s}=4$ causes a linear equation ( $\mathrm{RT}=949+4.2 \mathrm{~s})$ to fit the first responses better than a $\log$ function $\left(R T=830+58 \log _{2} s\right)$ does.

\section{CONCLUSIONS}

The results of the short series of Experiment I indicate that Sternberg's $(1966,1969)$ serial model of memory search holds as well for photographs of diverse scenes as it does for symbolic material. The search rate of $24 \mathrm{msec}$ per memory picture is faster than what one would expect from other experiments that used pictorial material as memory and probe items. For example, Sternberg (1969, pp. 493.441) reports a search rate of $56 \mathrm{msec} / \mathrm{item}$ for photographs of faces, and Klatzky and Atkinson (1971) obtained average search rates of about $70 \mathrm{msec} /$ item for drawings of familiar objects. It is possible that the color photographs used here were so diverse and easily discriminable that memory comparisons could be very brief and still be accurate.

Experiment II and the long series of Experiment I indicate, on the other hand, that the search rate for small memory loads does not continue for large ones. We should have predicted this from results of experiments on recognition memory for very large memory loads of pictures. For example. Wyant, Banks, Berger, and Wright (1972) used a two-alternative forced-choice task to test recognition with memory loads of 960 pictures. A search rate of $24 \mathrm{msec} /$ item would predict a mean RT of about $20 \mathrm{sec}$ for recognition (assuming, of course, that two-alternative forced choice would take as long as yes-no classification, but, if anything, it seems that the yes-no response would be faster). However, Ss almost always responded during the $10 \mathrm{sec}$ while the test stimuli were in view. We might also have predicted these results from those of Juola et al (1971), who found a very fast search rate for long memory sets.

Does the process of memory search change when memory load exceeds six items? Surely, if a serial model is to be maintained, its rate must change for large loads. The Atkinson-Juola model, which has small memory loads searched in a short-term store at a slow rate and large loads searched in a long-term store at a faster rate, does predict the trend of results in both of the present experiments. However, the longer latencies for error responses in larger memory sets in Experiment II raise at least minor problems for this model. A possible alternative to a two-store serial search model for these data is a parallel model that generates a negatively accelerated RT vs $s$ function. It is fair to say that the serial models have difficulty with these data mainly because they are very explicitly spelled out. A paralle! model that could account for the virtually linear RT vs $s$ 
functions for small $s$ and the negatively accelerated function as $s$ becomes large might prove very difficult to formulate.

\section{REFERENCES}

Atkinson, R. C., \& Juola, J. F. Factors influencing speed and accuracy of word recognition. In S. Kornblum (Ed.), Attention and performance IV. New York: Academic Press, 1973.

Juola. J. F., Fischler, I., Wood, C. T., \& Atkinson, R. C. Recognition time for information stored in long-term memory. Perception \& Psychophysics, 1971, 10, 8-14.

Klatzky, R. L., \& Atkinson, R. C. Specialization of the cerebral hemispheres in scanning for information in short-term memory. Perception \& Psychophysics, 1971, 10, 335-338.

Rapoport. A. A study of disjunctive reaction times. Behavioral Science, 1959, 4, 299-315.

Shepard, R. Recognition memory for words, sentences, and pictures. Journal of Verbal Learning \& Verbal Behavior, 1967, 6, 156-163.

Standing, L., Conezio, J., \& Haber, R. N. Perception and memory for pictures: Single-trial learning of 2500 visual stimuli. Psychonomic Science, 1970, 19, 73-74.

Sternberg, S. High-speed scanning in human memory. Science, $1966,153,652-654$.

Sternberg, S. Memory-Scanning: Mental processes revealed by reaction-time experiments. American Scientist, 1969, 57, 421-457.
Townsend, J. T. A note on the identifiability of parallel and serial processes. Perception \& Psychophysics, 1971, 10 , 161-163.

Welford. A. T. The measurement of sensory motor performance: Survey and reappraisal of twelve years' progress. Ergonomics, $1960,3,189-229$

Wingfield, A., \& Branca, A. A. Strategy in high-speed memory search. Journal of Experimental Psychology, 1970, 83, 63-67.

Wyant, S., Banks, W. P., Berger, D., \& Wright, P. W. Verbal and pictorial similarity in recognition of pictures. Perception \& Psychophysics, 1972, 12, 151-153.

\section{NOTE}

1. G. E. Briggs and A. M. Johnsen (Memory \& Cognition, $1973,1,91-100$ ) proposed a hybrid serial-parallel model of memory comparison that gives negatively accelerated search functions.
(Received for publication July 27, 1973; accepted July 28,1973 .) 